\title{
Effects of magnetospheric precipitation and ionospheric conductivity on the ground magnetic signatures of traveling convection vortices
}

\author{
L. Zhu, R. W. Schunk, and J. J. Sojka \\ Center for Atmospheric and Space Sciences, Utah State University, Logan
}

\begin{abstract}
By using an improved TCV model (Zhu et al., 1997), a quantitative study of the effects of magnetospheric precipitation and ionospheric background conductivity on the ground magnetic signatures of traveling convection vortices (TCVs) has been conducted. In this study the localized conductivity enhancement associated with the TCVs is present and the ratio of the Hall and Pedersen conductances vary both spatially and temporally according to the hardness of the TCV precipitation. It is found that a strong conductivity enhancement associated with hard TCV precipitation can significantly distort the TCV current closure in the ionosphere and lead to ground magnetic disturbance patterns with strong asymmetry in E-W direction. The asymmetry of the ground magnetic patterns is characterized by a stronger magnetic disturbance on the side of the upward field-aligned currents (clockwise convection cell) and a possible rotation of the whole magnetic patterns. Specifically, the modeling results predict that when the characteristic energy of the TCV precipitation is below $500 \mathrm{eV}$, the asymmetry of the ground magnetic patterns is minimal (less than $1 \%$ ) and may not be detectable. When the characteristic energy of the precipitation is about $7 \mathrm{keV}$, the asymmetry of the magnetic patterns can be well above $30 \%$. It is also found that a low ionospheric background conductivity favors the appearance of strong asymmetry in the ground magnetic patterns of the TCVs, while a high ionospheric background conductivity favors the appearance of strong ground magnetic disturbances but with less asymmetry. We concluded that the most favorable condition for the appearance of strong asymmetry in the TCV ground magnetic signatures is the condition of winter, solar minimum, and hard precipitation.
\end{abstract}

\section{Introduction}

Traveling convection vortices (TCVs) are the localized convection cells that appear in the prenoon or postnoon sectors of the high-latitude ionosphere and move in a predominantly antisunward direction. It has been realized that the TCVs are the ionospheric manifestations of the transient interactions between the solar wind and the magnetosphere and that they carry important information of the dynamics at the magnetopause. Because of the importance of the TCVs in Earth space environment, substantial research efforts have been made to study the characteristics of the TCVs and explore the specific generation mechanisms for the TCVs [Lanzerotti et al., 1986, 1991; Friis-Christensen et al., 1988; Glassmeier et al., 1989; Heikkila et al., 1989; Sibeck, 1990; McHenry et al., 1990a, b; Kivelson and Southwood, 1991; Glassmeier, 1992; Glassmeier and Heppner, 1992; Southwood and Kivelson, 1993; Vo et al., 1994; Lühr and Blawert, 1994; Bristow et al., 1995; Zesta et al., 1995; Sibeck, 1995; Sibeck et al., 1996; Sibeck and Korotova, 1996; Moretto et al., 1997].

The current systems of the TCVs can cause significant ground magnetic disturbances. Because the dynamical features of the TCVs are temporally transient with a short timescale and are also spatially localized, the ground magnetometer chains have been the most appropriate observational tools for the TCVs. The study

Copyright 1999 by the American Geophysical Union

Paper number 1998JA900175.

0148-0227/99/1998JA900175\$09.00 of the ground magnetic disturbances as well as their relationship to the TCV current structures has been the most important component of the TCV study to date.

Complementary to the observational study of ground magnetic signatures of the TCVs [e.g., Moretto et al., 1997], the theoretical modeling of the TCV ground magnetic signatures can help to systematically understand the relationship between the ground magnetic signatures and the TCV current structures and to provide testable theoretical predictions that can guide future observations. The results of theoretical modeling of ground magnetic disturbances associated with localized ionospheric current systems have been reported in several previous studies [McHenry and Clauer, 1987; Zhu and Kan, 1989; Wei and Lee, 1990; Chaston et al., 1993]. However, all these modeling studies mainly focused on the localized current systems associated with FTEs, and none of them have included the effects of conductivity enhancement and ground induction.

Zhu et al. [1997] developed a TCV model to specifically study the relationship between the TCV current structures and the associated ground magnetic disturbances in which both the effects of conductivity enhancement and ground induction are included. The importance of the effect of conductivity enhancement can be briefly summarized as follows: When the convection vortices of magnetospheric origin reach the jonosphere, the precipitation associated with the upward field-aligned currents of the TCVs can cause significant conductivity enhancement in a localized region. The localized conductivity gradient can then change the TCVs' current closure in the ionosphere and modify the TCVs' current system. On the other hand, the nonuniform conductivity 
invalidates the ideal situation in Fukushima's [1969] theorem, which says that if the conductance is uniform, the ground magnetic disturbance is produced exclusively by the Hall current. In the Zhu et al. [1997] model, the contributions from all three components of the TCV current system (Hall, Pedersen, and field-aligned currents) to ground magnetic disturbances are included since the conductivity is not uniform any more, which is different from all the previous work. Zhu et al.'s quantitative modeling results confirmed the importance of conductivity enhancement for the TCV current system and the associated ground magnetic disturbances and clearly showed that the localized conductivity enhancement can cause a significant distortion of the TCV current system and lead to a distortion of the ground magnetic disturbance patterns. They also found that the effect of induction currents on ground magnetic signatures of the TCVs is insignificant (less than 5\%).

\section{Effects of the Magnetospheric Precipitation and Ionospheric Background Conductivity}

Zhu et al.'s [1997] work was mainly to present the mathematical formulation of the TCV model and to examine the importance of the conductivity enhancement and induction currents for a set of typical magnetospheric and ionospheric conditions. Specifically, the average energy of the precipitating electrons associated with the upward field-aligned currents was assumed to be $1 \mathrm{keV}$ and the ionospheric conductivity condition was close to that of the summer solstice for all the runs shown in their work.

Observations show that the hardness of TCV precipitation varies over a wide range (from a few hundreds $\mathrm{eV}$ to a few $\mathrm{keV}$ ) and short-lived auroral brightenings can be associated with the TCVs [e.g., Heikkila et al., 1989; Potemra et al., 1990; Vo et al., 1994]. It is also shown by observations that the TCVs virtually occur at any time of the year and their ground magnetic signatures have strong seasonal dependence [e.g., Glassmeier et al., 1989]. Since the TCVs is an magnetosphere-ionosphere (MI) coupling process and the ionosphere is not just a passive load, the variation of hardness of the TCV precipitation can lead to the enhanced ionospheric conductivity with different characteristics (Hall conductivity versus Pedersen conductivity), which can then change the TCV current closure in the ionosphere. In turn, the different TCV ionospheric current systems can lead to different ground magnetic signatures of the TCVs.

In addition to the effect of the TCV precipitation described qualitatively in the above, the jonospheric background conductivity condition, which varies with the season and solar condition, can also influence the TCV ground magnetic signatures. Sibeck et al. [1996] found that the impulsive event occurrence patterns bear a striking resemblance to reported statistical patterns for the Hall conductivity and they suggested that the high-latitude distribution of the Hall conductivity may influence the signatures observed by ground magnetometers. Since they only considered the contribution of the Hall currents to the ground magnetic disturbances, a uniform conductivity assumption was used in their work. A further step along that direction can be the study of ionospheric conductivity effect on ground magnetic signatures when the localized nonuniform conductivity enhancement is present. The existence of the localized conductivity gradient caused by the TCV precipitation can make the situation quite different from those studied by Sibeck et al. [1996]. First, the mapping of the TCVs from the magnetosphere to the ionosphere is not a simple mapping any more and the ionosphere plays an active role to modify the magnetospheric current closure in the ionosphere as well as the return currents to the magnetosphere. Second, the large-scale background conductivity can influence the features of ground magnetic signatures via the locally enhanced conductivity. Since the TCVs is not a simple mapping process from the magnetosphere to the ionosphere due to the existence of the localized conductivity enhancement, this influence is not a simple linear one in which the intensity of ground magnetic disturbances just scales with the magnitude of the ionospheric background conductivity. For various ionospheric background conductivity conditions, the localized conductivity enhancement associated with a specific TCV magnetospheric driver can be quite different due to the different recombination rates in the ionosphere. The different localized conductivity enhancements can lead to different TCV ionospheric current structures and then lead to different ground magnetic signatures.

The primary goal of this paper is to quantitatively show the effects of magnetospheric precipitation and ionospheric background conductivity upon the ground magnetic signatures of the TCVs when the localized conductivity enhancement is included. To serve this purpose, Zhu et al. [1997] TCV model has been first improved to include the energy dependence of the ratio of the Hall and Pedersen conductivities, which is based on Robinson et al. [1987] work. In other words, the assumption of constant average energy used in Zhu et al. [1997] work has been removed and the average energy of the TCV precipitation as well as the ratio of the Hall and Pedersen conductivities vary both spatially and temporally in the present study. By using the improved TCV model, we systematically studied how the variations of TCV precipitation and large-scale ionospheric conductivity affect the ground magnetic signatures of the TCVs and the modeling results are presented in the following chapters.

\section{Modeling Results}

In our study, the ionosphere is assumed to be a slab with height-integrated conductivities and the Earth magnetic field lines are assumed to be perpendicular to the ionosphere. In the modeling, we focus on the TCVs occurring in the prenoon sector. Therefore the numerical domain is located in the morning sector of the high-latitude ionosphere. The numerical domain is 3600 $\mathrm{km}$ long in the E-W (x) direction and $1100 \mathrm{~km}$ wide in the N-S (y) direction. Actually, with some sign changes, the model can also be used to study the magnetic signatures of the TCVs occurring in the postnoon sector. A Cartesian coordinate system is adopted in the model in which the $\mathrm{x}$ axis points to the west, the $y$ axis points to the south, and the $\mathrm{z}$ axis is upward.

In the model, the convection vortices originating in the magnetosphere are externally imposed and are assumed to be voltage generators. Since the TCVs are due to the direct interactions between the solar wind and magnetosphere near the magnetopause, the solar wind inertia is sufficiently large so that the loading effect of the ionosphere on the TCV source regions should be insignificant on the zeroth order. This assumption could be violated in the inner magnetosphere where the plasma inertia is small (e.g., the plasma sheet). In such a situation, the loading of the ionosphere can make a noticeable modification of the magnetospheric convection. To precisely simulate the electrodynamics of the TCVs, one has to treat them in a timedependent M-I coupling system that further includes the self- 
consistent ionospheric current closure in the magnetospheric source regions and a dynamic magnetosphere, which is far beyond the scope of this paper.

To focus on the effects of the hardness of the TCV precipitation and the magnitude of the ionospheric background conductivity on the ground magnetic signatures of the TCVs, we turned off the effect of the induction currents in the model and ignored the gradient of large-scale background conductivity since it is small compared to the gradient of the locally enhanced conductivity caused by the TCV precipitation. Also, we use the same TCV speed of $4 \mathrm{~km} / \mathrm{s}$ for all cases since the effect of TCV speed variation on the ground magnetic signatures has been systematically studied in our previous work [Zhu et al., 1997] and such a speed was most commonly documented in the TCV observational studies [e.g., Friis-Christensen et al., 1988].

\subsection{Case 1: No Conductivity Enhancement (Base Case)}

To study the effects of precipitation and background conductivity when the localized conductivity enhancement is present, we need a base case in which the conductivity enhancement is excluded. Such a case is shown in Figure la, where the background ionospheric conductance corresponds to winter and solar minimum ( 2 mho) conditions, the TCV convection cells moves toward the nightside (positive $\mathbf{x}$ direction) with a constant speed of $4 \mathrm{~km} / \mathrm{s}$, and a clockwise convection cell (upward current) leads. The ratio of the background Hall and Pedersen conductance is 1 for this case and all the other cases shown in this work. Figure la (top) shows a pair of imposed magnetospheric convection cells moving westward. The potential difference between the two convection cells is $20 \mathrm{kV}$. Since the background conductivity is uniform and no conductivity enhancement associated with the TCV precipitation is included, the current closure of the TCVs in the ionosphere is symmetric.
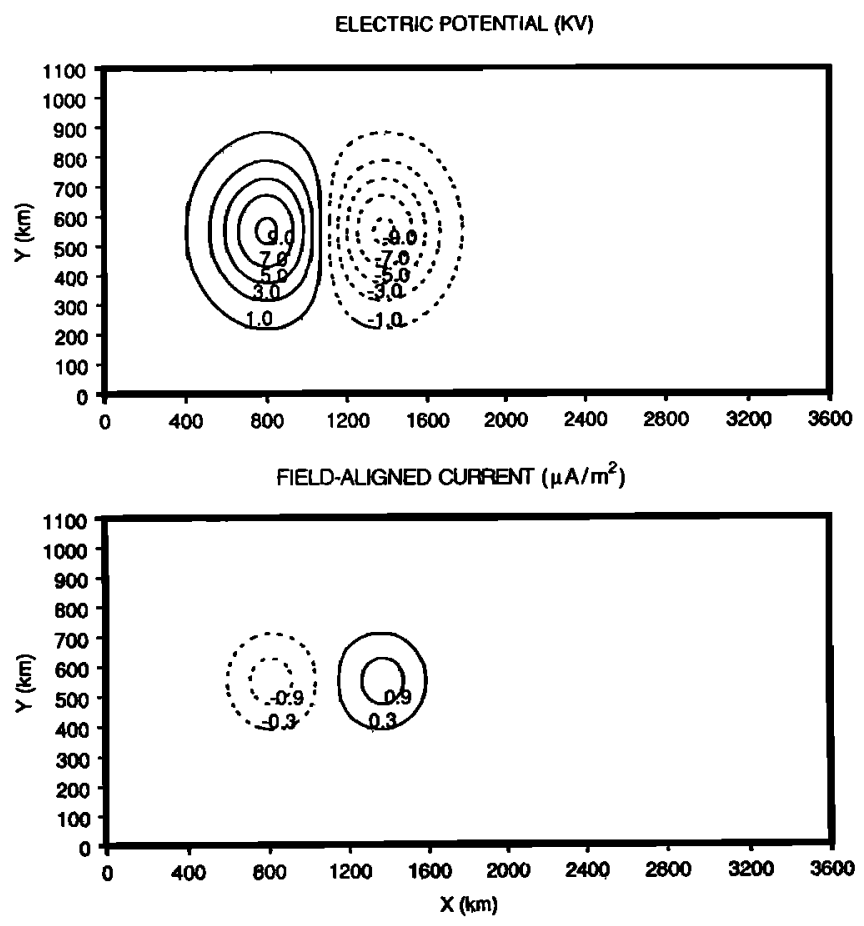

Figure 1a. (top) A pair of imposed magnetospheric convection cells moving westward and (bottom) the field-aligned currents associated with the TCVs. The solid lines in the field-aligned current plot represent the upward field-aligned currents.

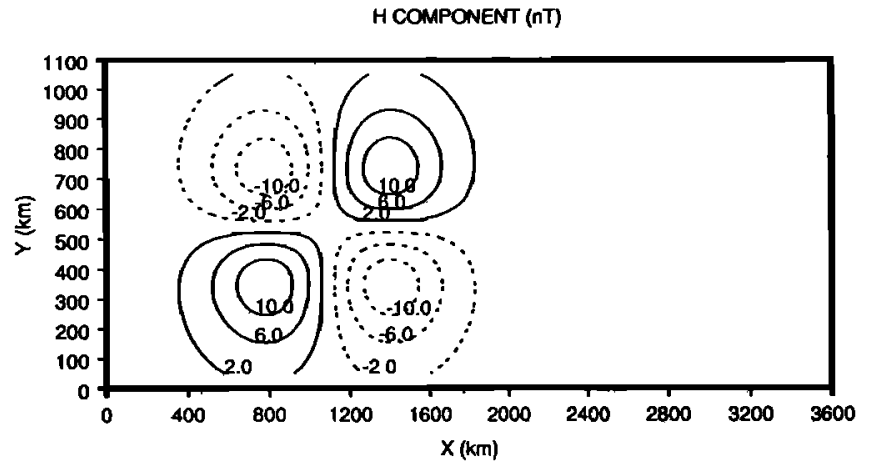

D COMPONENT (nT)

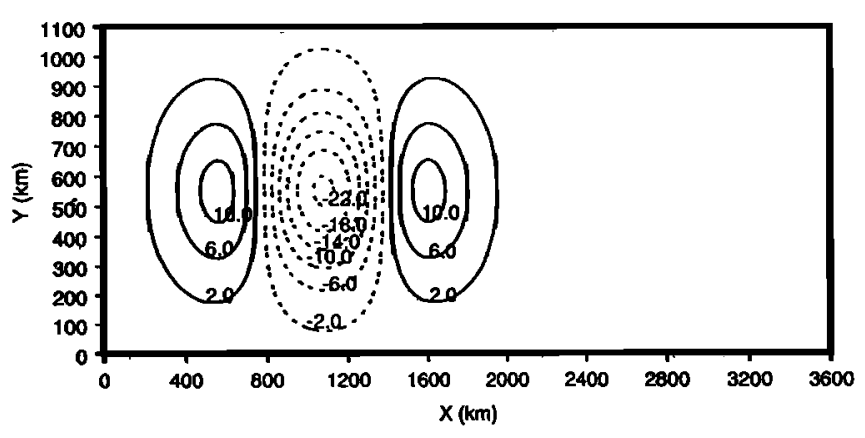

Z COMPONENT (nT)

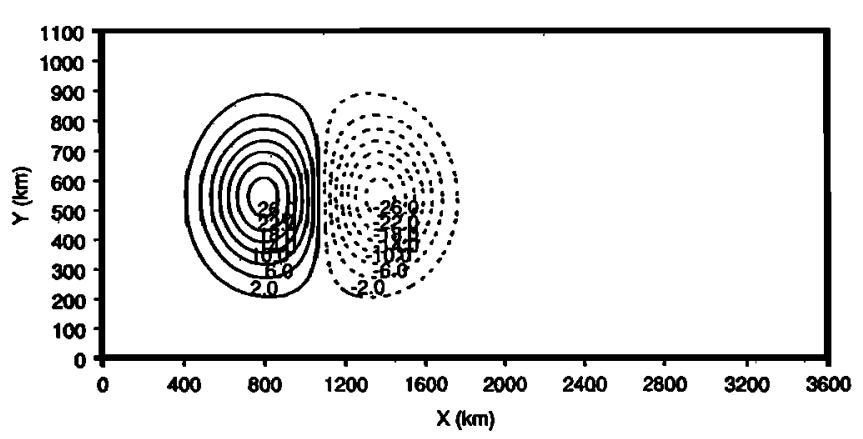

Figure 1b. Ground magnetic disturbance patterns of the (top) $\mathrm{H}$, (middle) $\mathrm{D}$, and (bottom) $\mathrm{Z}$ components associated with the TCVs in Figure la. In this case, the localized conductivity enhancement caused by the TCV precipitation is excluded.

A pair of symmetric field-aligned currents associated with the TCVs are shown in the bottom panel of Figure 1a, where solid lines represent the upward field-aligned currents. The maximum intensity of the field-aligned currents is about $1.1 \mu \mathrm{A} / \mathrm{m}^{2}$.

Figure $1 \mathrm{~b}$ shows the ground magnetic disturbance patterns of the $\mathrm{H}, \mathrm{D}$, and $\mathrm{Z}$ components associated with the TCVs. Note that even though the model includes the contributions from all three components of the TCVs' current system (Hall, Pedersen, and field-aligned currents) when it calculates the ground magnetic disturbances, the resulting magnetic patterns are the same as those associated with the Hall currents in this particular case. This is consistent with Fukushima's [1969] theorem. This equivalence will not hold when the ionospheric conductivity enhancement associated with the TCVs is included. The results shown in Figure $1 \mathrm{~b}$ indicate that when the conductivity is uniform the ground magnetic disturbance patterns are all symmetric for a pair of externally imposed symmetric convection cells. The 
maximum intensities of the $\mathrm{H}, \mathrm{D}$, and $\mathrm{Z}$ components in this case are 12,22 , and $28 \mathrm{nT}$, respectively.

For all the other cases shown in the following, we included the localized conductivity enhancement, thus we can study the effects of precipitation and background conductivity when the locally enhanced conductivity is present. It should be noted that even though the numerical modeling in this study is time dependent, in the following, we only show the results when the features of the localized conductivity enhancement approach asymptotic steady states.

\subsection{Case 2: Hard Precipitation, Low Conductivity}

Figure 2a shows the case in which the background ionospheric conductivity condition (winter and solar minimum) and the characteristics of the TCV convection cells (upward current cell leads and a speed of $4 \mathrm{~km} / \mathrm{s}$ ) are the same as those of case 1 , but the localized conductivity enhancement caused by the TCV precipitation is included. The peak characteristic energy of the precipitation associated with the TCVs is about $7.8 \mathrm{keV}$, which is
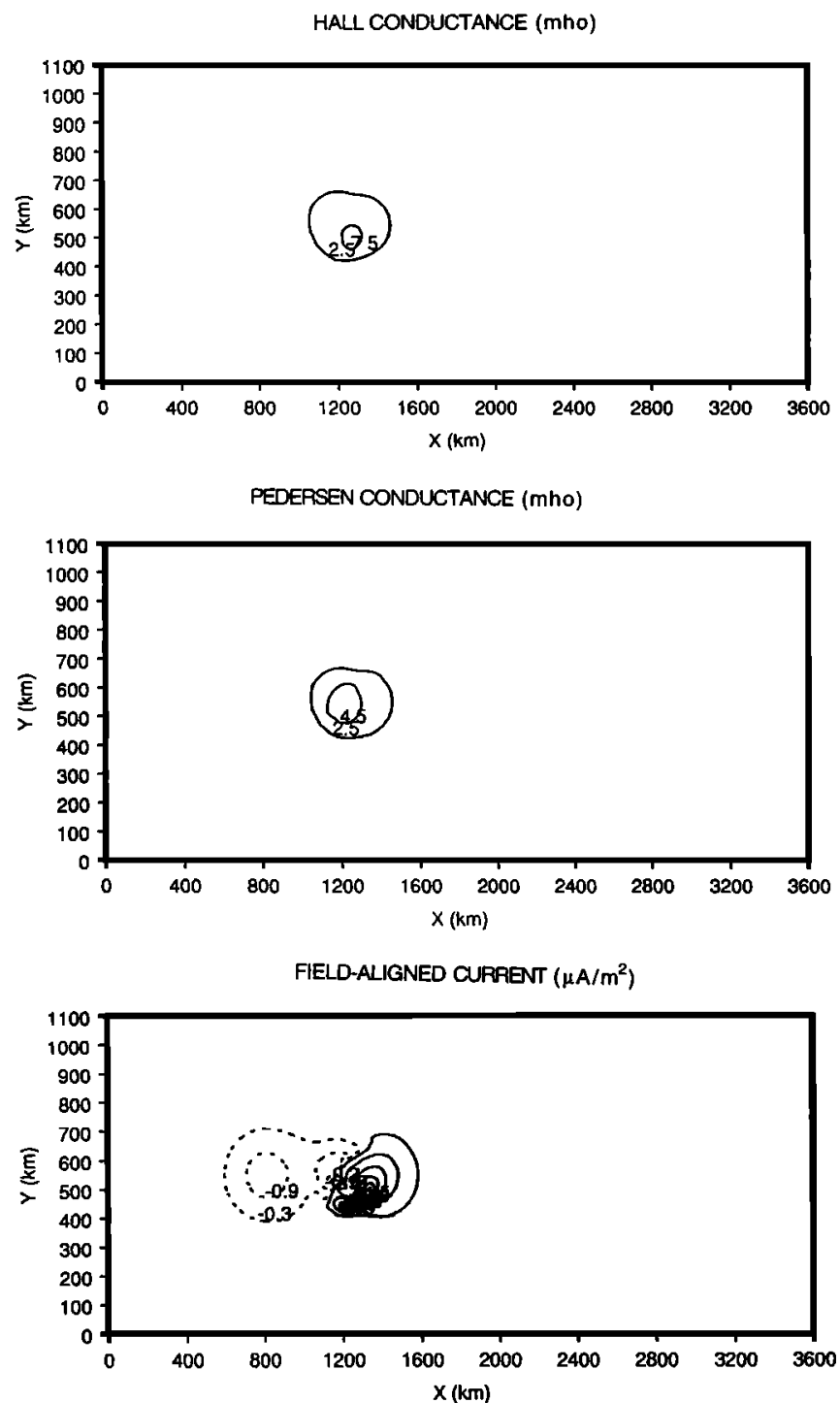

Figure 2a. Localized (top) Hall and (middle) Pedersen conductance enhancements for the case of hard TCV precipitation (peak characteristic energy is $7.8 \mathrm{keV}$ ); (bottom) distorted fieldaligned current distribution.

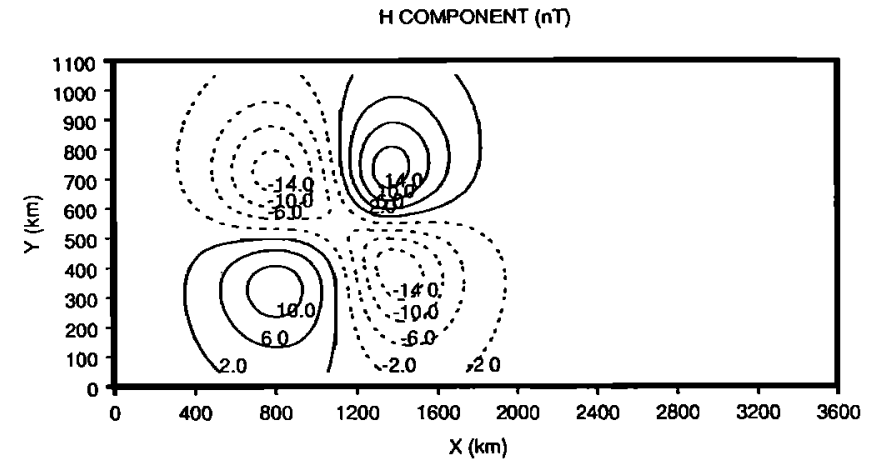

D COMPONENT (nT)

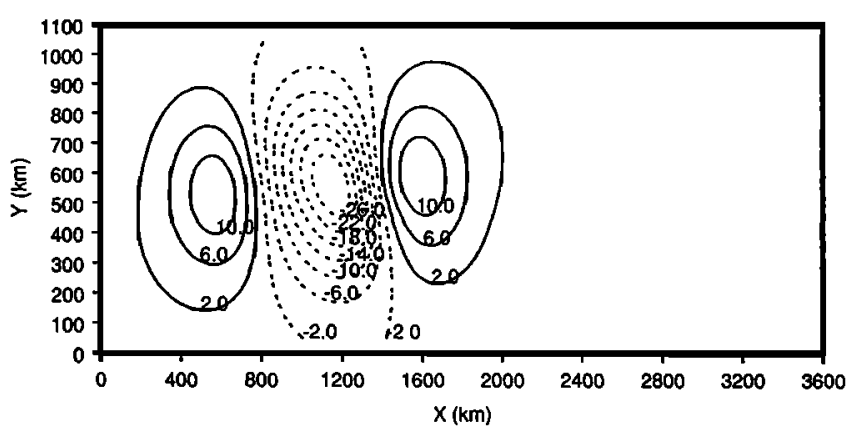

$z$ COMPONENT (nT)

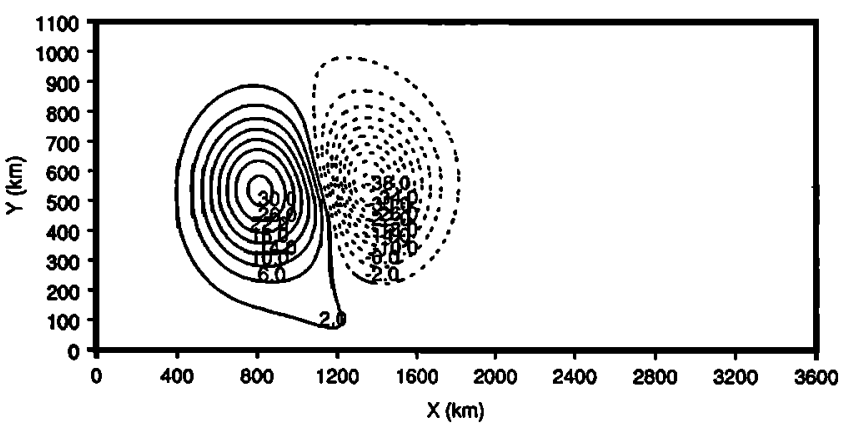

Figure 2b. Ground magnetic disturbance patterns of the (top) $\mathrm{H}$, (middle) $\mathrm{D}$, and (bottom) $\mathrm{Z}$ components associated with the TCVs with hard precipitation.

considered as the hard precipitation case in this study. The choice of the parameters for this case as well as those for all other cases are based on the TCV observations [e.g., Heikkila et al., 1989; Friis-Christensen et al., 1988] and the goal of this theoretical modeling study.

Figure 2a (top) shows the localized Hall conductance enhancement caused by the TCV precipitation and the middle panel shows the distribution of the Pedersen conductance. Since we only consider the ionization due to the energetic precipitating electrons associated with upward currents, the resulted single localized high conductivity region is expected. Because of the jonization effect of the TCV precipitation, the Hall conductance in the upward current region increases to $9.3 \mathrm{mho}$ and the Pedersen conductance increases to $6.1 \mathrm{mho}$. Compared to the background conductances ( $2 \mathrm{mho}$ ), the Hall conductance has increased by a factor of 4.6 and the Pedersen conductance has increased by a factor of 3 for this hard precipitation case.

Because of the conductivity gradient, the current closure of the TCVs in the ionosphere is no longer symmetric and both the 
horizontal and field-aligned currents are distorted. The distorted field-aligned current distribution is shown in Figure 2 a (bottom). As can be seen, the current distortion caused by the enhanced conductivity is most significant on the upward current cell since the ionization effect associated with the precipitation in the - downward current cell is not significant and has been ignored in the model. Because the conductivity gradient changes the TCVs' magnetospheric current closure in the ionosphere, a localized downward current cell appears at the southeast edge of the leading convection cell. It can be seen that the larger downward current cell associated with the counterclockwise convection cell is also distorted, but the distortion is not as severe as that of the upward current cell. Because of the highly enhanced conductance caused by the hard precipitation, the upward currents are greatly intensified and the maximum intensity of the upward currents reaches about $4.9 \mu \mathrm{A} / \mathrm{m}^{2}$ in this case, which is about 3.5 times larger than that of the downward field-aligned currents.

It needs to be mentioned that there is a significant dislocation (about $100 \mathrm{~km}$ ) between the regions of the maximum Hall and Pedersen conductances shown in Figure 2a, but the regions of the maximum Hall conductance and the maximum upward fieldaligned currents are quite close. This is because hard precipitation is most effective in increasing the Hall conductance and the hard precipitation is spatially colocated with strong upward field-aligned currents in the model.

Figure $2 b$ shows the ground magnetic disturbance patterns of the $H, D$, and $Z$ components. It is found that for this hard precipitation case, the maximum intensities of the $H$ and $D$ components of the magnetic disturbances have increased $30 \%$ from those of case 1 , while that of the $\mathrm{Z}$ component has increased $36 \%$. Since the ionospheric conditions and the convection cells as well as the associated precipitation are the same for both cases, the intensity increases of the ground magnetic disturbances must be due to the localized conductivity enhancement. A close look at the magnetic disturbance patterns in Figure $2 \mathrm{~b}$ shows that the intensity increases of the magnetic disturbances caused by the conductivity enhancement actually lead to asymmetries of the patterns, which is characterized by stronger magnetic disturbances on the side of the upward field-aligned currents. Obviously, these asymmetries of the ground magnetic patterns are related to the asymmetrically enhanced conductivity, which mainly occurs in the regions of the upward field-aligned currents. Another feature that leads to further asymmetries of the magnetic patterns shown in Figure $2 b$ is a noticeable counterclockwise rotation of the patterns. Such a rotation of the patterns is related to the conductivity enhancement caused by the hard precipitation, which in turn leads to distortions of the TCVs' current systems. A detailed discussion on the cause of the magnetic pattern rotation will be given after we present the results of case 5 in which a downward current cell leads instead of the upward current cell in this case.

These asymmetries of the TCV magnetic disturbance patterns caused by strong conductivity enhancement associated with hard precipitation should be observable with break ground magnetometers. The model results suggest that the predicted asymmetries should be most noticeable in the $\mathrm{Z}$ component of magnetogram. For the specific TCVs with hard precipitation shown in Figure 2a, a magnetometer near the center of the TCVs should observe a $30 \%$ asymmetry in the $\mathrm{Z}$ component record. For the magnetometers away from the TCV center, these asymmetries in the $\mathrm{Z}$ component will increase even though the magnitudes of the $\mathrm{Z}$ component disturbances decrease. The difference of the predicted asymmetries in the $\mathrm{Z}$ components for magnetometers southward of the TCVs and northward of the TCVs is that the former will observes a stronger $Z$ component disturbance followed by a weaker $Z$ component disturbance with an opposite sign and the latter will observe a weaker $Z$ component disturbance followed by a stronger $Z$ component disturbance. These predicted features of ground magnetogram for the TCVs are, of course, related to the asymmetrically intensified ground magnetic disturbances and the rotation of the magnetic disturbance patterns predicted by the model.

\subsection{Case 3: Soft Precipitation, Low Conductivity}

To further study the effects of hardness of the TCV precipitation on the ground magnetic signatures, we considered a case in which the background ionospheric conductivity condition (winter and solar minimum) and the polarity (upward fieldaligned currents lead) and speed $(4 \mathrm{~km} / \mathrm{s})$ as well as the spatial scale of the TCVs are the same as those of case 2, but the precipitation is much softer (peak characteristic energy is about

HAL CONDUCTANCE (mho)

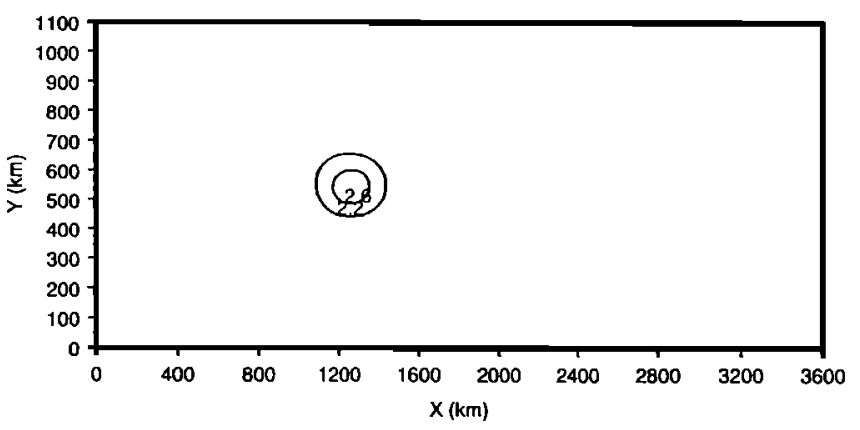

PEDERSEN CONDUCTANCE (mho)

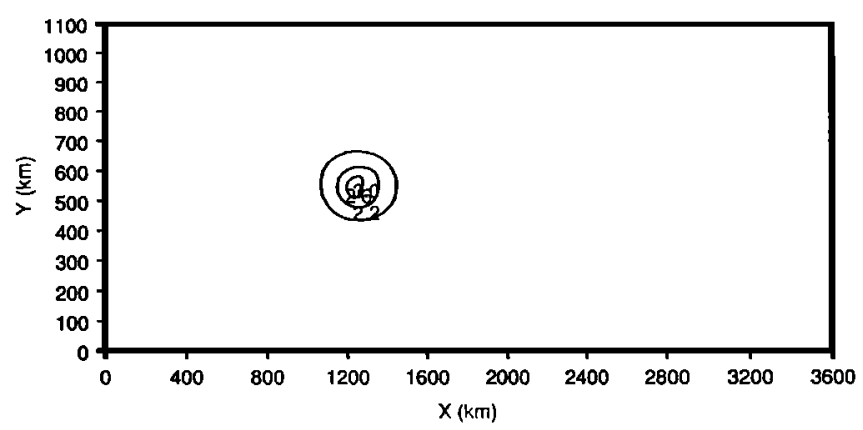

FIELD-ALIGNED CURRENT $\left(\mu A / \mathrm{m}^{2}\right)$

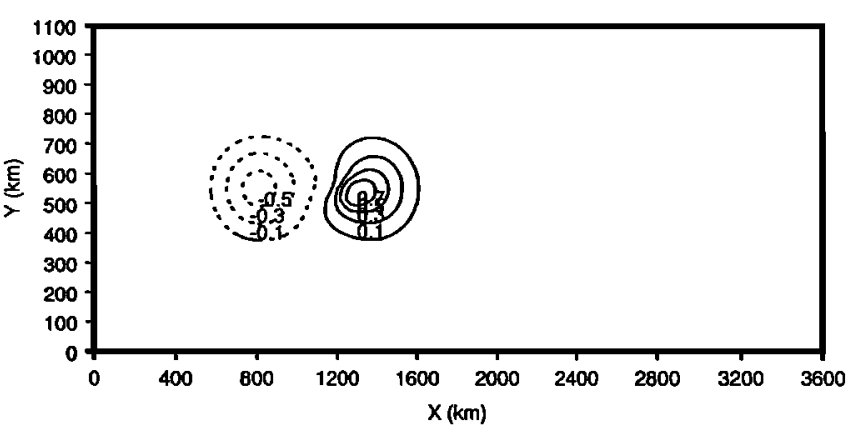

Figure 3a. Localized (top) Hall and (middle) Pedersen conductance enhancements as well as the (bottom) field-aligned current distribution for the case of soft TCV precipitation (peak characteristic energy is $1.3 \mathrm{keV}$ ). 
H COMPONENT (nT)

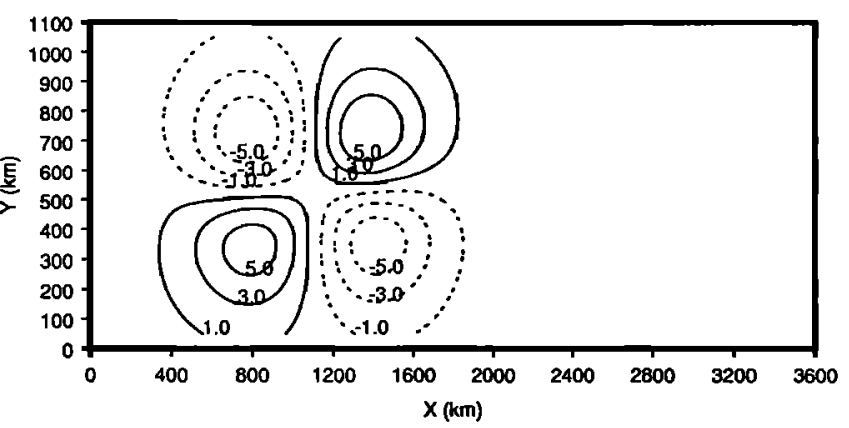

D COMPONENT (nT)

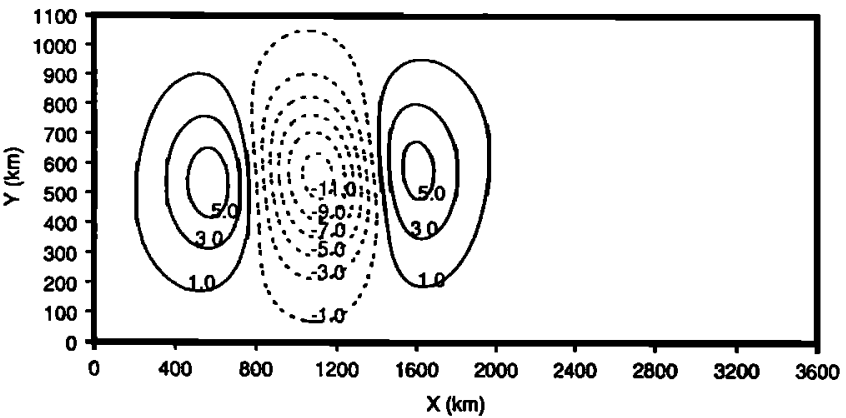

$Z$ COMPONENT (nT)

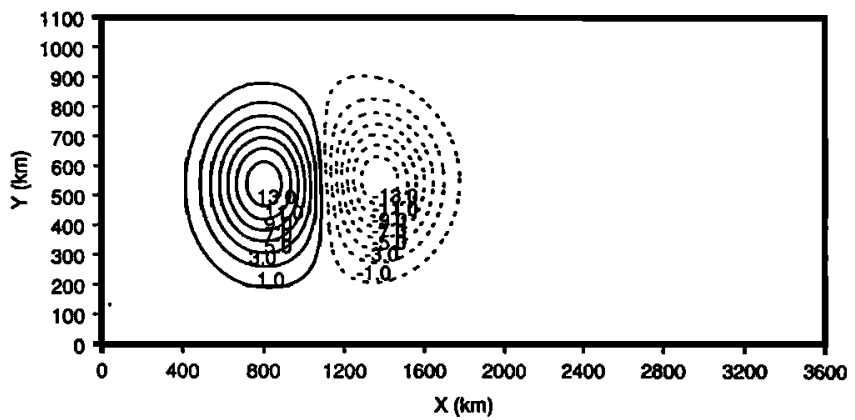

Figure 3b. Ground magnetic disturbance patterns of the (top) $H$, (middle) $\mathrm{D}$, and (bottom) $\mathrm{Z}$ components associated with the TCVs with soft precipitation.

$1.3 \mathrm{keV}$ ). To self-consistently achieve such a soft precipitation, the potential difference between two convection cells has been reduced to $10 \mathrm{keV}$ instead of $20 \mathrm{keV}$ as in case 1 and 2 .

Figure 3a (top) shows the localized Hall conductance enhancement and the middle panel shows the Pedersen conductance. Because the precipitation in this case is much softer than that of case 2 , the Hall conductance only increases to 2.9 mho and the Pedersen conductance increases to 3.1 mho. The small ratio $(0.93)$ of the Hall and Pedersen conductances is consistent with the softness of the precipitation. Compared to Figure $2 \mathrm{a}$, it can be seen that the distortion of the field-aligned currents (shown in Figure 3a (bottom)) caused by conductivity enhancement is noticeable in this case but not as significant as that of the hard precipitation case. The comparison also shows a total disappearance of the localized downward current cell at the edge of the clockwise convection cell for the soft precipitation case. This is because the conductivity gradient in this soft precipitation case is small, otherwise a large conductivity gradient will force the magnetospheric upward currents to be closed locally to form a localized downward current cell. Such localized field-aligned current filaments caused by large conductivity gradient associated with hard precipitation have also been reported to occur in the substorm aurora regions [e.g., Zhu and $K a n, 1990]$. The maximum intensity of the upward currents for this soft precipitation case is about $0.8 \mu \mathrm{A} / \mathrm{m}^{2}$.

Figure $3 \mathrm{~b}$ shows the ground magnetic disturbance patterns of the $\mathrm{H}, \mathrm{D}$, and $\mathrm{Z}$ components for the case of soft precipitation. It can be seen that the magnitude asymmetry of the magnetic patterns is very small (about $2 \%$ ). Also, the rotation of the whole magnetic patterns is much smaller than that of the hard precipitation case. We ran a case of extremely soft precipitation (peak characteristic energy is $500 \mathrm{eV}$ ) (not shown here) and found the asymmetry of the ground magnetic patterns is below $1 \%$. On the basis of these model results, a theoretical prediction useful to the TCV observations could be that an asymmetry of less than $1 \%$ on magnetogram may indicate the characteristic energy of the TCV precipitation is well below $1 \mathrm{keV}$, while an asymmetry of more than $30 \%$ may indicate the TCVs with a characteristic energy of above $7 \mathrm{keV}$.

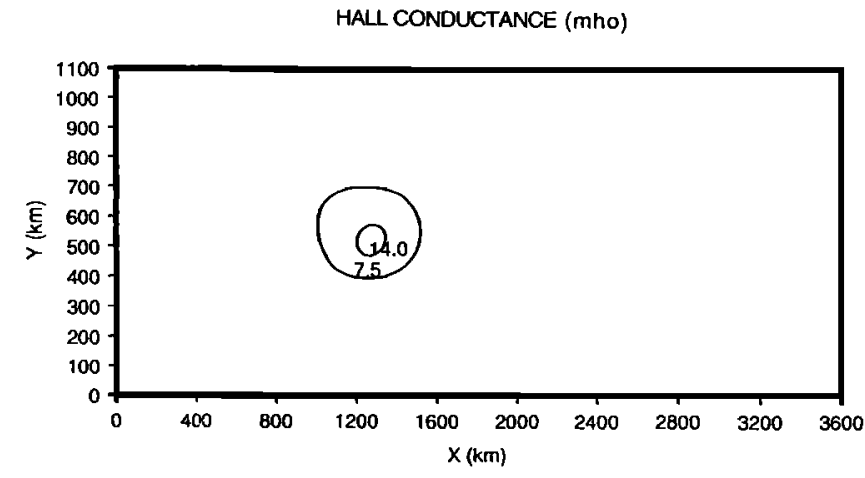

PEDERSEN CONDUCTANCE (mho)

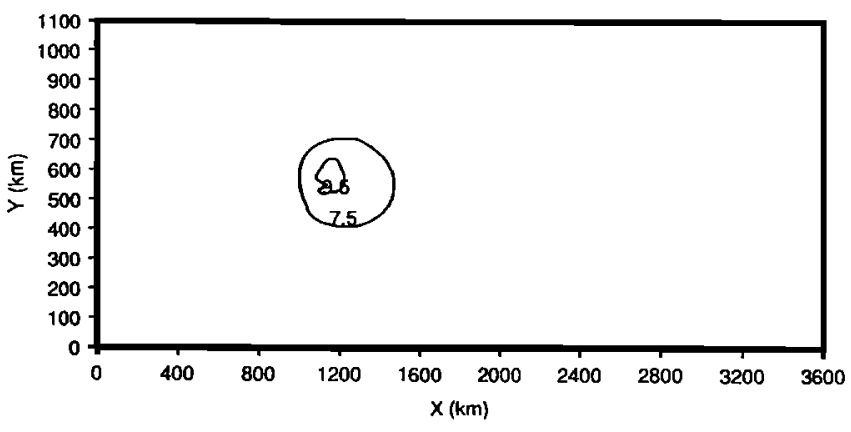

FIELD-ALIGNED CURRENT $\left(\mu \mathrm{A} / \mathrm{m}^{2}\right)$

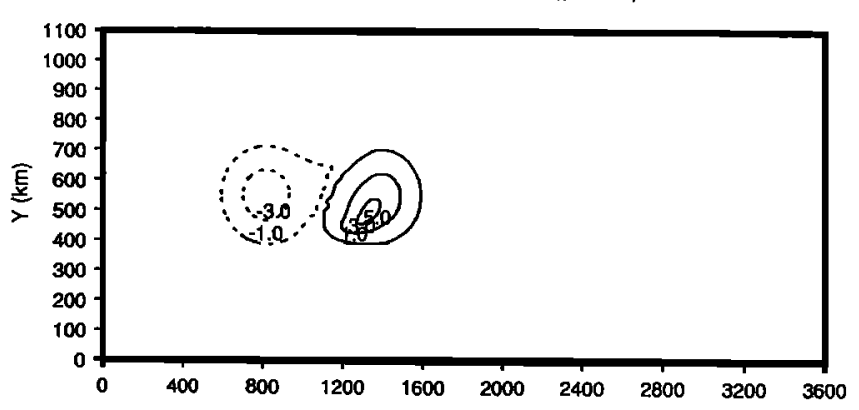

Figure 4a. Localized (top) Hall and (middle) Pedersen conductance enhancements as well as the (bottom) field-aligned current distribution for the case of high ionospheric background conductivity (summer and solar maximum). 


\subsection{Case 4: Hard Precipitation, High Conductivity}

As we discussed in the above, the large-scale background conductivity can influence the features of the TCV ground magnetic signatures via a locally enhanced conductivity and this influence is not a simple scaling effect. To quantitatively study such an effect, we ran a case in which the TCV convection and precipitation are the same as those of case 2 , but the ionospheric background conductivity conditions change from the winter and solar minimum ( 2 mho) to the summer and solar maximum ( 7 mho). Then we made the comparison of this case and case 2 .

Figure 4a (top) shows the Hall conductance enhancement while the middle panel shows the distribution of the Pedersen conductance. The Hall conductance in the upward current region increases to 15 mho from the background level of 7 mho and the Pedersen conductance increases to $10 \mathrm{mho}$. In other words, the Hall and Pedersen conductances are enhanced by factors of 2.1 and 1.4 , respectively, which are much smaller than those for the case of winter and solar minimum (4.6 and 3). A higher uniform background conductivity and a smaller localized conductivity enhancement relative to the background level in this case

H COMPONENT (nT)

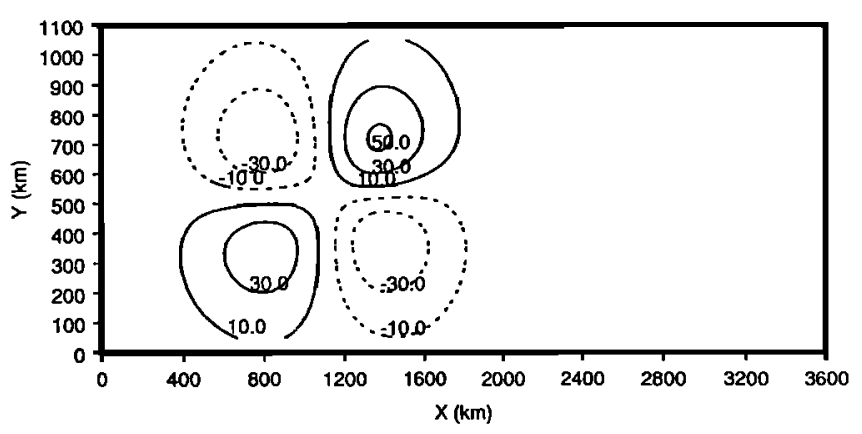

D COMPONENT (nT)

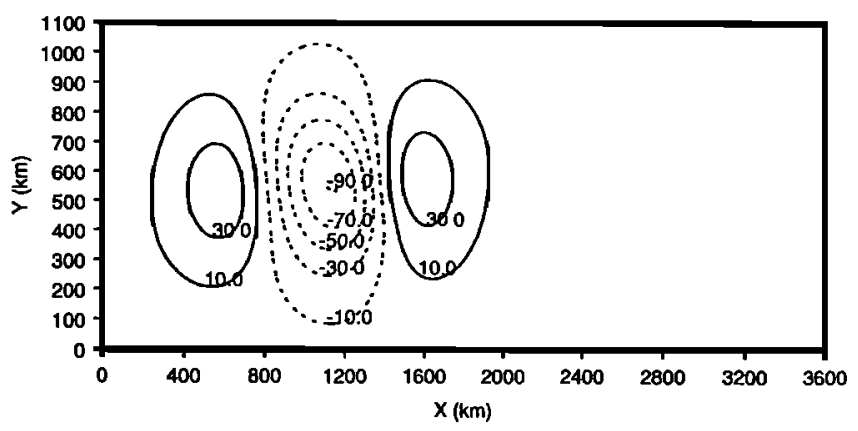

$Z$ COMPONENT (nT)

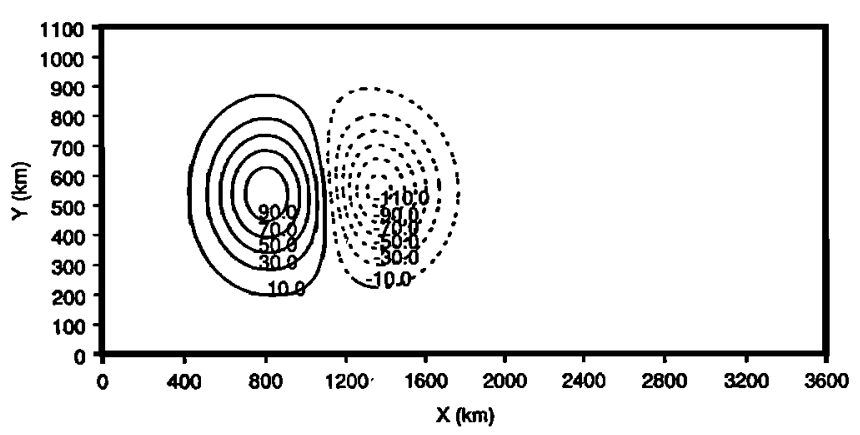

Figure 4b. Ground magnetic disturbance patterns of the (top) $H$, (middle) $\mathrm{D}$, and (bottom) $\mathrm{Z}$ components associated with the TCVs for summer and solar maximum conditions. determine that the distortion of the TCVs' current system must be smaller than that for the winter and solar minimum (case 2). This feature can be seen clearly from the comparison of the fieldaligned current distributions in Figures $2 \mathrm{a}$ and $4 \mathrm{a}$.

Figure $4 \mathrm{~b}$ shows the ground magnetic disturbance patterns of the $H, D$, and $Z$ components for the case of summer and solar maximum. Since the TCVs' current system is less distorted, the ground magnetic disturbance patterns show less asymmetries than those for the winter and solar minimum conditions. The model results indicate that a magnetometer near the center of the TCVs can only observe a $12 \%$ asymmetry in the $\mathrm{Z}$ component record for this specific case of summer and solar maximum, while it will be $30 \%$ for the winter and solar minimum with the same TCV convection and precipitation. Also, the rotations of the magnetic patterns are much smaller for the summer and solar maximum than those for the winter and solar minimum, even though the TCV magnetospheric convections and precipitations are the same.

\subsection{Case 5: Downward Current Cell Leading}

For the case of hard precipitation (case 2), there is a noticeable rotation of the ground magnetic patterns, while for the case of soft precipitation the pattern rotation is much smaller. This indicate the hardness of precipitation can influence the features of the ground magnetic pattern rotation. However, what is the physics behind it? How does the hardness of precipitation affect the rotation of the ground magnetic patterns? To answer these questions, we ran a case (case 5) in which the TCV convection and ionospheric conditions are the same as those in case 2 except for the polarity of the convection cells being switched. Now we have a leading downward field-aligned current cell instead of a leading upward field-aligned cell as in case 2.

The modeling results of case 5 are shown in Figures $5 \mathrm{a}$ and $5 \mathrm{~b}$. Compared the results of case 2 and case 5 , it can be seen that the conductivity enhancements are largely the same for both cases, but the downward field-aligned current cell in case 5 is almost undisturbed and symmetric. This is because that the TCVs' convection cells move toward nightside (positive $\mathrm{x}$ direction) and the regions of the enhanced conductivity associated with the upward field-aligned currents (clockwise convection cell) are always behind the downward field-aligned current cell (counterclockwise convection cell). Therefore the downward field-aligned current cell will not be disturbed by the enhanced conductivity.

A more interesting feature of this case can be seen in the ground magnetic disturbance patterns shown in Figure 5b. These is a total disappearance of the rotation of magnetic patterns. Since the only difference between case 2 and case 5 is the change of convection cell polarity, comparison of the two cases can provide a physical explanation for the cause of the ground magnetic pattern rotations, which is briefly described in the following. When the TCV convection vortices of magnetospheric origin reach the ionosphere, a significant conductivity enhancement can be caused by the precipitation associated with the upward field-aligned currents of the TCVs. In turn, the upward currents and their closure in the ionosphere can be distorted by the localized conductivity enhancement. For the TCVs with a leading upward current cell, the high speed of the TCVs allows a significant portion of the downward current cell to intrude into the enhanced conductivity region left by the upward current cell before the conductivity enhancement subsides due to the ionospheric recombination. Thus the downward field-aligned current cell can also be distorted by the conductivity gradient. 


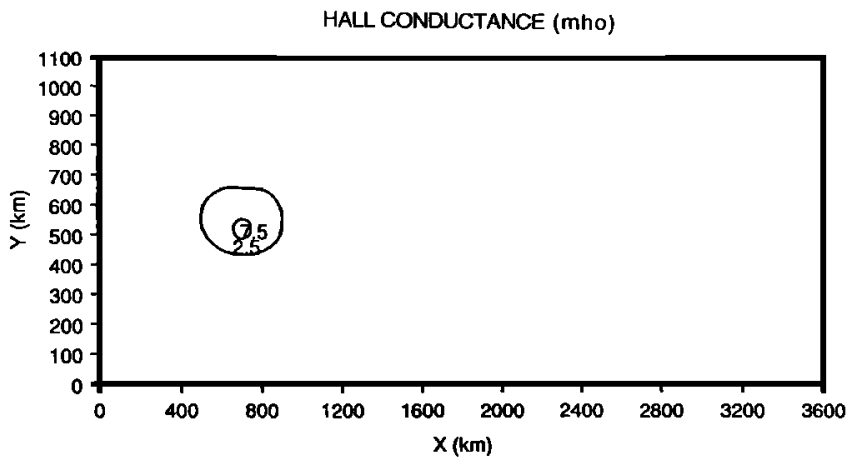

PEDERSEN CONDUCTANCE (mho)
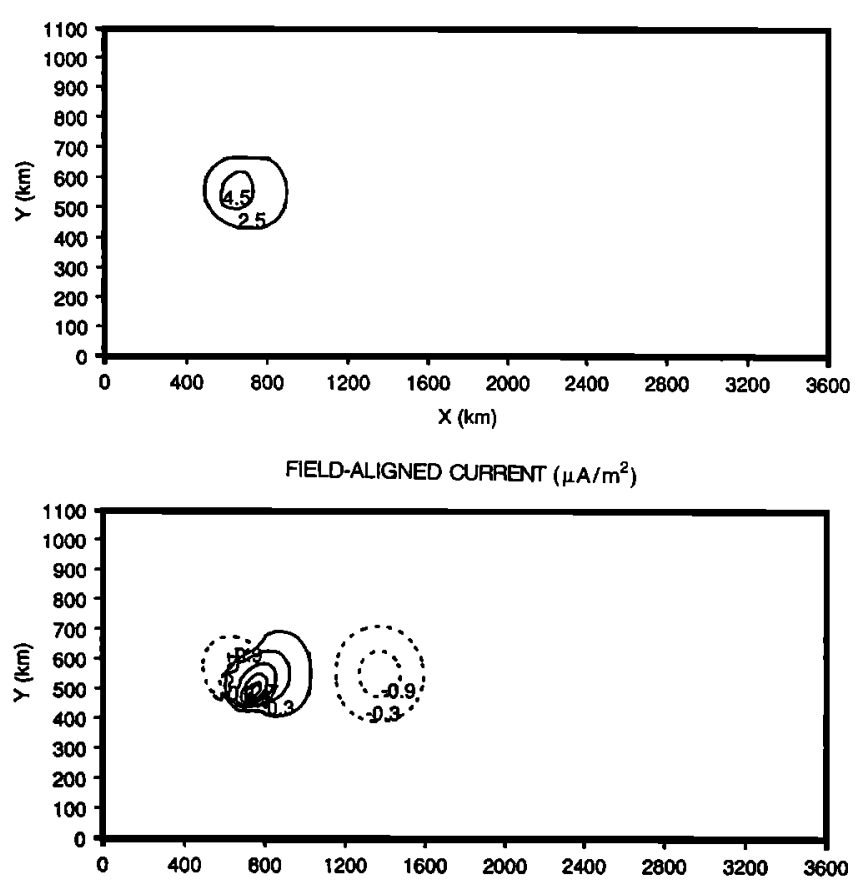

Figure 5a. (top) Hall and (middle) Pedersen conductance distributions as well as the (bottom) field-aligned current distribution for the case of the TCVs with a leading downward current cell (leading counterclockwise convection cell).

The distortions of both the field-aligned current cells can then lead to a rotation of the whole ground magnetic patterns. The situation for the TCVs with a leading downward current cell can be quite different. Since the enhanced conductivity region is always behind the downward current cell, only the upward current cell is distorted and there will be no rotation of the whole magnetic patterns. The next question is what is the influence of the hardness of precipitation in such a physical process? The answer to this is now quite simple. Hard precipitation can cause a stronger conductivity enhancement and a larger conductivity gradient, which can lead to more significant distortion of both the field-aligned current cells for the TCVs with a leading upward current cell. A greater distortion of both the field-aligned current cells will lead to a larger rotation of the TCV ground magnetic patterns. This physical picture is consistent with the modeling results of case 2 and case 3 , in which the hardness of precipitation and the rotation of the magnetic patterns have a positive correlation.

\section{Summary}

By úsing an improved TCV model [Zhu et al., 1997], a quantitative study of the effects of magnetospheric precipitation and ionospheric background conductivity on the ground magnetic signatures of the TCVs has been conducted, in which the localized conductivity enhancement associated with the TCVs is included. The major results are summarized as follows:

1. A strong conductivity enhancement associated with hard TCV precipitation can significantly distort the TCV current closure in the ionosphere and lead to ground magnetic disturbance patterns with strong asymmetry in E-W direction. The pattern of the $\mathrm{Z}$ component shows the strongest asymmetry.

2. The E-W asymmetry of the TCV ground magnetic patterns can consist of two parts. One is a stronger disturbance on the side of the upward current cell (clockwise convection cell) and the other is a rotation of the whole magnetic patterns. Both parts of the asymmetry increase when the hardness of the TCV precipitation increases, but the latter disappears for the TCVs with a leading downward current cell (counterclockwise convection cell) no matter how hard the TCV precipitation is.
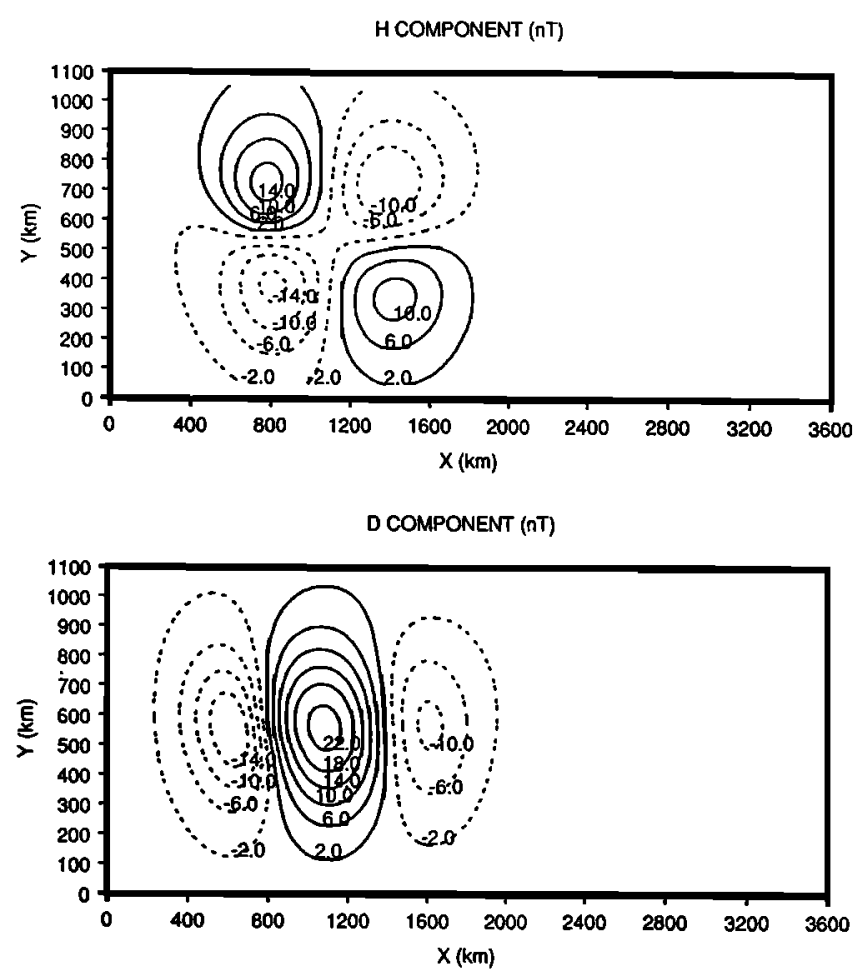

$Z$ COMPONENT ( $\mathrm{nT})$

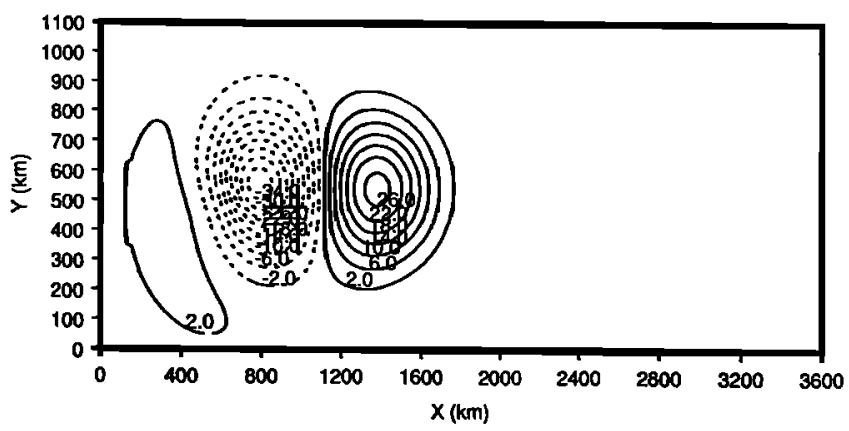

Figure 5b. Ground magnetic disturbance patterns of the (top) $H$, (middle) $\mathrm{D}$, and (bottom) $\mathrm{Z}$ components associated with the TCVs with a leading counterclockwise convection cell. 
3. The modeling results predict that when the characteristic energy of the TCV precipitation is below $500 \mathrm{eV}$, the asymmetry of the ground magnetic patterns is minimal (less than $1 \%$ ) and may not be detectable. When the characteristic energy of the precipitation is about $7 \mathrm{keV}$, the asymmetry of the magnetic patterns can be well above $30 \%$.

4. One of the model predictions which is especially useful for analyzing ground magnetogram data from latitudinal chain stations is that whenever two stations observe a latitudinal asymmetry such that one magnetogram shows a stronger $Z$ component disturbance followed by a weaker $Z$ component with an opposite sign and the other shows a weaker $Z$ component followed by a stronger $Z$ component with an opposite sign, a rotation of ground magnetic patterns as well as the TCVs with a leading downward current cell must exist. Such a latitudinal asymmetry becomes stronger when the TCV precipitation is harder.

5. A low ionospheric background conductivity favors the appearance of strong asymmetry in the ground magnetic patterns of the TCVs, while a high ionospheric background conductivity favors the appearance of strong ground magnetic disturbances but with less asymmetry.

6. The most favorable condition for the appearance of strong asymmetry in the TCV ground magnetic signatures is that of winter, solar minimum, and hard precipitation. For such a condition, the E-W asymmetry of the 2-D Z component patterns can go beyond $35 \%$, while the asymmetry of the 1-D cut observed by a magnetometer away from the center of the TCVs can be as great as $60 \%$.

Since the observed magnetic disturbance patterns normally appear to be distorted and asymmetric [Friis-Christensen et al., 1988], the quantitative results of the 2-D ground magnetic patterns with asymmetric features shown in this work can be useful for deriving the electrodynamical structures of the TCVs from the magnetogram data of ground magnetometer chains. To proceed in that direction, a follow up work could be a one-to-one comparison study between the model results and observational data in which we can determine whether the cause of the observed asymmetries of ground magnetic disturbances is the localized conductivity enhancement associated with the TCV precipitation. To serve that purpose, magnetic data from the high-latitude magnetometer chains, for example, CANOPUS, AGO, Scandinavian and Greenland chains, will be most useful.

Acknowledgment. This research was supported by NASA grant NAG5-1484, and NSF grants ATM-96-12835 and ATM-96-12638 to Utah State University.

Janet G. Luhmann thanks A. V. Kustov and Frederick Menk for their assistance in evaluating this paper.

\section{References}

Bristow, W. A., et al., Observations of convection vortices in the afternoon sector using the SuperDARN HF radars, J. Geophys. Res., $100,19743,1995$

Chaston, C. C., H. J. Hansen, F. W. Menk, B. J. Fraser, and Y. D. Hu, Ground signatures of convecting reconnected flux tubes, J. Geophys. Res., 98, 19151, 1993.

Friis-Christensen, E., M. A. McHenry, C. R. Clauer, and S. Vennerstrom, Ionospheric traveling convection vortices observed near the polar cleft: A triggered response to sudden changes in the solar wind, Geophys. Res. Lett., 15, 253, 1988.

Fukushima, N., Equivalence in ground geomagnetic effect of ChapmanVestine's break and Birkland-Alfvén's electric current-systems for polar magnetic storms, Rep. Ionos. Space Res. Jpn., 23(3), 219, 1969.

Glassmeier, K., Traveling magnetospheric convection twin-vortices: Observations and theory, Ann. Geophys., 10,547, 1992.

Glassmeier, K., and C. Heppner, Traveling magnetospheric convection twin vortices: Another case study, global characteristics and a model, J. Geophys. Res., 97, 3977, 1992.

Glassmeier, K., M. Honisch, and J. Untiedt, Ground-based and satellite observations of traveling magnetospheric convection twin vortices, $J$. Geophys. Res., 94, 2520, 1989.

Heikkila, W. J., T. S. Jorgensen, L. J. Lanzerotti, and C. G. Maclennan, A transient auroral event on the dayside, J. Geophys. Res., 94, 15291, 1989.

Kivelson, M. G., and D. J. Southwood, Ionospheric traveling vortex generation by solar wind buffeting of the magnetosphere, J. Geophys. Res., 96, 1661, 1991.

Lanzerotti, L. J., L. C. Lee, C. G. Maclennan, A. Wolfe, and L. V. Medford, Possible evidence of flux transfer events in the polar ionosphere, Geophys. Res. Lett., I3, 1089, 1986.

Lanzerotti, L. J., R. M. Konik, A. Wolfe, D. Venkatesan, and C. G. Maclennan, Cusp latitude magnetic impulse events, 1, Occurrence, statistics, J. Geophys. Res., 96, 14009, 1991.

Lühr, H., and W. Blawert, Ground signatures of traveling convection vortices, in Solar Wind Sources of Magnetospheric Ultra-Low-Frequency Waves, Geophys. Monogr. Ser., vol. 81, edited by M. J. Engebretson, K. Takahashi, and M. Scholer, p. 231, AGU, Washing ton, D.C., 1994.

McHenry, M. A., and C. R. Clauer, Modeled ground magnetic signatures of flux transfer events, J. Geophys. Res., 92, 11,231, 1987.

McHenry, M. A., C. R. Clauer, E. Friis-Christensen, P. T. Newell, and J. D. Kelly, Ground observations of magnetospheric boundary layer phenomena, J. Geophys. Res., 95, 14995, 1990a.

McHenry, M. A., C. R. Clauer, and E. Friis-Christensen, Relationship of solar wind parameters to continuous dayside, high latitude traveling ionospheric convection vortices, J. Geophys. Res., 95, 15007, 1990b.

Moretto, T., E. Friis-Christensen, H. Luhr, and E. Zesta, Global perspective of ionospheric traveling convection vortices: Case studies of two Geospace Environmental Modeling events, J. Geophys. Res., 102, 11597, 1997.

Potemra, T. A., H. Vo, D. Venkatesan, L. L. Cogger, R. E. Erlandson, L. J. Zanetti, P. F. Bythrow, and B. J. Anderson, Periodic auroral form and geomagnetic field oscillations in the $1400 \mathrm{MLT}$ region, J. Geophys. Res., 95, 5835, 1990.

Robinson, R. M., R. R. Vondrak, K. Miller, T. Dabbs, and D. Hardy, On calculating ionospheric conductances from the flux and energy of precipitating electrons, J. Geophys. Res., 92, 2565, 1987.

Sibeck, D. G., A model for the transient magnetospheric response to sudden solar wind dynamic pressure variations, J. Geophys. Res., 95, $3755,1990$.

Sibeck, D. G., Current systems associated with magnetic impulse events and traveling convection vortices (abstract), Eos Trans. AGU, 76 (46), Fall Meet. Suppl., F489, 1995.

Sibeck, D. G., and G. I. Korotova, Occurrence patterns for transient magnetic field signatures at high latitudes, J. Geophys. Res., I0I, 13413, 1996.

Sibeck, D. G., R. A. Greenwald, W. A. Bristow, and G. I. Korotova, Concerning possible effects of ionospheric conductivity upon the occurrence patterns of impulsive events in high-latitude ground magnetograms, J. Geophys. Res., I0I, 13407, 1996.

Southwood, D. J., and M. G. Kivelson, Vortex motion in the ionosphere and nonlinear transport, J. Geophys. Res., 98, $11459,1993$.

Vo, H. B., J. S. Murphree, R. D. Elphinstone, R. C. Elphic, E. FriisChristensen, H. Luhr, and D. G. Sibeck, Multipoint observations of a dayside transient event, J. Geophys. Res., 99, 13409, 1994.

Wei, C. Q., and L. C. Lee, Ground magnetic signatures of moving elongated plasma clouds, J. Geophys. Res., 95, 2405, 1990.

Zesta, E., W. J. Hughes, and M. J. Engebretson, The evolution of traveling convection vortices (TCV's) (abstract), Eos Trans. AGU, 76 (46), Fall Meet. Suppl., F516, 1995.

Zhu, L., and J. R. Kan, Ground magnetic signatures of multiple fieldaligned current sheets in flux transfer events, J. Geophys. Res., 94, $6655,1989$.

Zhu, L., and J. R. Kan, Effects of ionospheric recombination time scale on the auroral signatures of substorms, J. Geophys. Res., 95, 10389, 1990.

Zhu, L., P. Gifford, J. J. Sojka, and R. W. Schunk, Model study of ground magnetic signatures of traveling convection vortices, J. Geophys. Res., $102,7449,1997$.

R. W. Schunk, J. J. Sojka, and L. Zhu, Center for Atmospheric and Space Sciences, Utah State University, Logan, UT 84322-4405. (zhu@cc.usu.edu)

(Received May 4, 1998; revised November 10, 1998;

accepted December 16, 1998.) 